\title{
LÖWENHEIM-SKOLEM AND INTERPOLATION THEOREMS IN INFINITARY LANGUAGES ${ }^{1}$
}

\author{
BY DAVID W. KUEKER \\ Communicated by F. W. Gehring, September 15, 1971
}

Let $L$ be a first-order finitary predicate language with equality. For each pair of infinite cardinals $\kappa$ and $\lambda$ with $\kappa \geqq \lambda$ we let $L_{\kappa \lambda}$ be the logic extending $L$ which allows the conjunction $(\wedge)$ and disjunction $(\vee)$ of fewer than $\kappa$ formulas and the simultaneous universal or existential quantification of fewer than $\lambda$ variables. We set $L_{\infty \lambda}=\bigcup_{\kappa} L_{\kappa \lambda}$. The standard syntactical and semantical concepts are defined as usual (see [1], [2]). If $\theta$ is a sentence we write $\mathfrak{U} \vDash \theta$ to mean that $\theta$ is true on the model $\mathfrak{U}$. $\mathfrak{A} \equiv_{{ }_{k \lambda}} \mathfrak{B}$ means that $\mathfrak{A}$ and $\mathfrak{B}$ have the same true sentences of $L_{\kappa \lambda} \cdot \mathfrak{A}, \mathfrak{B}$, and $\mathfrak{I}_{i}$ are always used for models for $L$, and we follow the convention that their universes are $A, B, A_{i}$ respectively. The cardinality of a set $X$ is denoted by $|X|$. If $L^{\prime}$ is some other language, then $L_{\kappa \lambda}^{\prime}$ is the corresponding infinitary logic built on $L^{\prime}$. For ease in stating many of our results we assume, except in the last section, that $L$ has only countably many nonlogical symbols. A detailed presentation of these and related results is in preparation for publication elsewhere.

1. $L_{\infty \omega}$ and the Löwenheim-Skolem theorem. One form of the downward Löwenheim-Skolem theorem for sentences of $L_{\omega_{1} \omega}$ can be stated as follows:

(A) If $\mathfrak{U} \vDash \theta$, then $\mathfrak{A}_{0} \vDash \theta$ for some countable $\mathfrak{U}_{0} \subseteq \mathfrak{A}$. The conclusion of $(\mathrm{A})$ is quite weak ; certainly the converse does not generally hold. One of our first goals is to define a notion of "almost all" such that the following biconditional holds for sentences of $L_{\omega_{1} \omega}$ :

(B) $\mathfrak{U} \vDash \theta$ iff $\mathfrak{U}_{0} \models \theta$ for almost all countable $\mathfrak{U}_{0} \subseteq \mathfrak{A}$. More importantly, we also generalize (B) to apply to sentences of $L_{\infty \omega}$ (for which (A) usually fails). To do this we must first index the countable submodels of a model and define countable approximations to any sentence of $L_{\infty \omega \omega}$.

Let $\kappa$ be an uncountable cardinal. We define a filter $D$ over $\mathscr{P}_{\omega_{1}}(\kappa)$, the countable subsets of $\kappa$, as follows:

Definition. $X \subseteq \mathscr{P}_{\omega_{1}}(\kappa)$ belongs to $D$ iff $X$ contains some $X^{\prime}$ such that (i) for every $s \in \mathscr{P}_{\omega_{1}}(\kappa)$ there is some $s^{\prime} \in X^{\prime}$ such that $s \subseteq s^{\prime}$ and (ii) $X^{\prime}$ is closed under unions of countable chains.

LEMMA. $D$ is a countably complete filter, and if $X_{\xi} \in D$ for all $\xi<\kappa$ then $\left\{s: s \in X_{\xi}\right.$ for all $\left.\xi \in s\right\} \in D$.

\footnotetext{
AMS 1970 subject classifications. Primary $02 \mathrm{H} 10,02 \mathrm{~B} 20,02 \mathrm{~B} 25$.
${ }^{1}$ Research supported in part by the National Science Foundation under grant NSF GP 20298 .
} Copyright (C) American Mathematical Society 1972 
Definition. Let $\mathfrak{A}$ be a model with $|A| \leqq \kappa$. Let $A=\left\{a_{\xi}: \xi<\kappa\right\}$. If $s \in \mathscr{P}_{\omega_{1}}(\kappa)$ we define $\mathfrak{X}_{s}$ to be the submodel of $\mathfrak{A}$ generated by $\left\{a_{\xi}: \xi \in s\right\}$.

Terminology. "For almost all $s$ " means "for all $s$ in some set belonging to D." "For almost all countable submodels of $\mathfrak{A}$ " means "for $\mathfrak{A}_{\boldsymbol{s}}$ for almost all $s . "$

REMARKS. (1) $\mathfrak{A}_{s}$ is almost independent of enumeration of the elements of $A$; that is, if $A=\left\{a_{\xi}^{\prime}: \xi<\kappa\right\}$ then $\left\{a_{\xi}: \xi \in s\right\}=\left\{a_{\xi}^{\prime}: \xi \in s\right\}$ for almost all $s$. "Almost all countable submodels of $\mathfrak{A}$ " therefore has a definite meaning independent of the cardinal $\kappa \geqq|A|$ and the enumeration of $A$.

(2) The filter $D$ has a game-theoretic characterization. If $X \subseteq \mathscr{P}_{\omega_{1}}(\kappa)$ we define the game $G_{x}$ played as follows: I and II alternately choose elements of $\kappa$; I wins if the resulting set of their choices belongs to $X$, and II wins otherwise. Then I has a winning strategy for $G_{x}$ iff $X \in D$.

For the next definition we assume that the formulas of a conjunction or disjunction in $L_{\kappa+\omega}$ are indexed by $\kappa$.

DEFINITION. Let $\theta$ be a formula of $L_{\kappa+\omega}$. We define its approximations $\theta^{s}$ for $s \in \mathscr{P}_{\omega_{1}}(\kappa)$ by induction:

(i) if $\theta$ is atomic then $\theta^{s}$ is $\theta$;

(ii) if $\theta$ is $\neg \psi(\exists x \psi, \forall x \psi)$ then $\theta^{s}$ is $\neg \psi^{s}\left(\exists x \psi^{s}, \forall x \psi^{s}\right)$;

(iii) if $\theta$ is $\wedge_{\xi<\kappa} \theta_{\xi}\left(\vee_{\xi<\kappa} \theta_{\xi}\right)$ then $\theta^{s}$ is $\wedge_{\xi \in S} \theta^{s}\left(\vee_{\xi \in S} \theta_{\xi}^{s}\right)$.

Notice that $\theta^{s}$ is always a formula of $L_{\omega_{1} \omega}$, and that if $\theta$ is a formula of $L_{\omega_{1} \omega}$, then $\theta^{s}$ is $\theta$ for almost all $s$.

By induction on formulas, using the Lemma giving properties of $D$, we obtain the following generalized Löwenheim-Skolem theorem.

THEOREM 1. Assume that $|A| \leqq \kappa$, and let $\theta$ be a sentence of $L_{\kappa+\omega}$. Then $\mathfrak{U} \vDash \theta$ iff $\mathfrak{U}_{s} \vDash \theta^{s}$ for almost all $s$.

As immediate consequences we obtain result (B) above and the following :

Corollary. Assume $\theta$ can be written in negation-normal form (that is, only atomic subformulas are negated) without uncountable disjunctions. Then $\vDash \theta$ iff $\vDash \theta^{s}$ for almost all s. In particular, if $\sigma$ and $\psi_{\xi}(x)$ belong to $L_{\omega_{1} \omega}(\xi<\kappa)$, then $\vDash \sigma \rightarrow \exists x \wedge_{\xi<\kappa} \psi_{\xi}(x)$ iff $\vDash \sigma \rightarrow \exists x \wedge_{\xi \in s} \psi_{\xi}(x)$ for all countable $s \subseteq \kappa$.

Another consequence of Theorem 1 is the following characterization of $\equiv_{\infty \omega}$ which generalizes Scott's Isomorphism Theorem (see [1]).

Theorem 2. Assume that $|A|,|B| \leqq \kappa$. Then

(i) $\mathfrak{U} \equiv_{\infty \omega} \mathfrak{B}$ iff $\mathfrak{U}_{s} \cong \mathfrak{B}_{s}$ for almost all $s$;

(ii) $\mathfrak{U} \not_{\infty \omega} \mathfrak{B}$ iff $\mathfrak{A}_{s} \not \mathfrak{B}_{s}$ for almost all $s$. 
To prove Theorem 2 it is enough to prove both of the implications from left to right. For (i) this is not difficult, using the standard back-and-forth properties of $\equiv_{\infty \omega}$ (see [1]). For (ii) this is immediate from Theorem 1. S. Shelah has observed that (ii) also follows from a game-theoretic characterization of $\equiv_{\infty \omega}$ and the Gale-Stewart theorem that open games are determined.

As might be expected from Theorem 2, reduced products of countable models modulo the filter $D$ can also be used to characterize $\equiv_{\infty \omega}$.

DEFINITION. (a) $L^{*}$ is the expansion of $L$ formed by adding a new predicate $P_{\neg}$ for every predicate $P$ (including $=$ ) of $L$. (b) If $\mathfrak{A}$ is an $L$-model then $\mathfrak{U}^{*}$ is its expansion to $L^{*}$ satisfying

$$
\forall v_{0} \cdots v_{k}\left[P \neg\left(v_{0}, \cdots, v_{k}\right) \leftrightarrow \neg P\left(v_{0}, \cdots, v_{k}\right)\right] .
$$

(c) If $\mathfrak{U}^{\prime}$ is an $L^{*}$-model and $\mathfrak{B}^{\prime} \subseteq \mathfrak{U}^{\prime}$, then $\mathfrak{B}^{\prime}$ is strongly maximal in $\mathfrak{U}^{\prime}$ if $B^{\prime}$ is a maximal subset of $A^{\prime}$ satisfying $\forall x y(x=\neg y \leftrightarrow \neg x=y)$.

THEOREM 3. Assume that $|A|,|B| \leqq \kappa$. The following are equivalent:

(i) $\mathfrak{Y} \equiv{ }_{\infty \omega} \mathfrak{B}$;

(ii) $\Pi \mathfrak{U}_{s}^{*} / D \cong \Pi \mathfrak{B}_{s}^{*} / D$;

(iii) $\mathfrak{B}^{*}$ is isomorphic to a strongly maximal submodel of $\Pi \mathfrak{U}_{s}^{*} / D$.

The implication from (i) to (ii) is immediate from Theorem 2(i). The implication from (ii) to (iii) is not difficult, using the Lemma giving properties of $D$. To show that (iii) implies (i) we first show that $\left\{s: \mathfrak{U}_{s} ¥ \mathfrak{B}_{s}\right\} \notin D$, and then use Theorem 2 (ii) to conclude that $\mathfrak{A} \equiv_{\infty \omega \omega} \mathfrak{B}$. Examples show the * is necessary for (ii) to imply (i).

We also obtain results analogous to Theorems 2 and 3 for embeddability in place of isomorphism.

\section{2. $L^{p}(\omega)$ and closed classes.}

DEFINITION. Let $\boldsymbol{K}$ be a class of models closed under isomorphism.

(a) $\boldsymbol{K}$ is closed if: $\mathfrak{A} \in \boldsymbol{K}$ iff $\mathfrak{A}_{0} \in \boldsymbol{K}$ for almost all countable $\mathfrak{U}_{0} \subseteq \mathfrak{U}$.

(b) $\boldsymbol{K}$ is closed downward if: whenever $\mathfrak{A} \in \boldsymbol{K}$, then $\mathfrak{U}_{0} \in \boldsymbol{K}$ for almost all countable $\mathfrak{U}_{0} \subseteq \mathfrak{U}$.

Classes which are closed downward satisfy a downward LöwenheimSkolem theorem, while closed classes also satisfy an upward theorem. Theorem 1 implies that $\operatorname{Mod}(\sigma)$ is closed if $\sigma$ is a sentence of $L_{\omega_{1} \omega}$, and Theorem 2 implies that closed classes are closed under $\equiv_{\infty \omega}$. A closed class is uniquely determined by the countable models in it, and hence, there are $2^{2 \omega}$ different closed classes. If $K$ and its complement are closed downward then $\boldsymbol{K}$ is closed, but the converse fails. If $\boldsymbol{K}^{\prime}$ is a class of $L^{\prime}$-models which is closed downward, then $K^{\prime} \uparrow L$ (=the class of all reducts of models in $\boldsymbol{K}^{\prime}$ to $\left.L\right)$ is also closed 'downward. Therefore $\operatorname{Mod}\left(\sigma^{\prime}\right) \uparrow L$ is closed downward, but not generally closed, for any sentence $\sigma^{\prime}$ of $L_{\omega_{1} \omega}^{\prime}$. 
We define $L^{p}(\omega)$ to be the class of formulas of Keisler's $L(\omega)$ (from [2]) which can be put in prenex form. Thus, $\sigma \in L^{p}(\omega)$ iff $\sigma$ is equivalent to some $\left(Q_{n} v_{n}\right)_{n<\bar{\omega}} \chi$, where $\chi$ is a quantifier-free formula (in countably many variables). Therefore $\sigma \in L^{p}(\omega)$ if $\sigma$ is a formula of $L_{\omega_{1} \omega}$ or a universal or existential sentence of $L_{\omega_{1} \omega_{1}}$. If $\sigma$ is a sentence of $L^{p}(\omega)$ then $\operatorname{Mod}(\sigma)$ is closed downward, but not generally closed. Most of the interest of $L^{p}(\omega)$ stems from:

THEOREM 4. If $\boldsymbol{K}$ is closed then $\boldsymbol{K}=\operatorname{Mod}(\sigma)$ for some sentence $\sigma$ of $L^{p}(\omega)$.

COROLLARY 1. If $\boldsymbol{K}$ is closed downward then there is a sentence $\sigma$ of $L^{p}(\omega)$ such that $\boldsymbol{K} \subseteq \operatorname{Mod}(\sigma)$, and $\boldsymbol{K}$ and $\operatorname{Mod}(\sigma)$ contain precisely the same countable models.

The intersection of two classes which are closed downward is either empty or contains a countable model. Hence, Corollary 1 implies a separation result for disjoint classes closed downward, a particular case of which is the following interpolation theorem for $L^{p}(\omega)$.

COROLLARY 2. Let $L_{1}$ and $L_{2}$ be countable languages whose intersection is $L$. Let $\theta \in L_{1}^{p}(\omega)$ and $\psi \in L_{2}^{p}(\omega)$, and assume that $\vDash \theta \rightarrow \neg \psi$. Then there is some $\sigma \in L^{p}(\omega)$ such that $\vDash \theta \rightarrow \sigma$ and $\vDash \sigma \rightarrow \neg \psi$.

The case of Corollary 1 where $K=\operatorname{Mod}(\bar{\Sigma}) \nmid L$ for some set $\bar{\Sigma}$ of finitary sentences is due to Svenonius [3]. Corollary 2 is essentially due to Takeuti (see the next section). Even if $\theta$ and $\psi$ are also in $L_{i_{\omega_{1} \omega_{1}}}$, Malitz's example (given in [4]) shows that the interpolant $\sigma$ need not be in $L_{\infty \omega_{1}}$.

The logic $L^{p}(\omega)$ also admits some preservation theorems.

Definition. (a) $\mathfrak{A}$ is the $\beta$-union of a nonempty set $S$ of submodels of $\mathfrak{A}$ (where $\beta$ is any cardinal $>0$ ) if every subset of $A$ of power less than $\beta$ is contained in the universe of some model in $S$.

(b) $\left(\forall^{n} \exists\right)^{p}(\omega)$ is the set of all sentences of $L^{p}(\omega)$ of the form $\forall x_{0} \cdots x_{n-1}$ $\exists y_{0} \cdots y_{k} \cdots \chi$, where $\chi$ is quantifier-free.

THEOREM 5. (i) $K$ is closed downward and closed under $(n+1)$-unions iff $K=\operatorname{Mod}(\theta)$ for some $\theta \in\left(\forall^{n} \exists\right)^{p}(\omega)$.

(ii) $\boldsymbol{K}$ is closed downward and closed under $\omega$-unions iff $\boldsymbol{K}=\operatorname{Mod}\left(\wedge_{n} \theta_{n}\right)$ where $\theta_{n} \in\left(\forall^{n} \exists\right)^{p}(\omega)$ for all $n$.

A sentence whose negation is in $\left(\forall^{0} \exists\right)^{p}(\omega)$ is universal. Case $n=0$ of Theorem 5 then implies: $\boldsymbol{K}$ is closed and closed under submodels iff $\boldsymbol{K}=\operatorname{Mod}(\theta)$ for some universal $\theta$ of $L^{p}(\omega)$. This is a different formulation of a theorem of Tarski [5].

3. Generalizations to uncountable models. If $\lambda$ is any infinite cardinal and $\kappa>\lambda$ we can define a filter over $\mathscr{P}_{\lambda}^{+}(\kappa)$ analogously to $\S 1$ and 
obtain a notion of "almost all" subsets of $\kappa$ of power at most $\lambda$. Most of the results of the preceding sections have analogues here, especially if $\lambda^{\lambda}=\lambda$. Some of them are of less interest, however, due to the failure of the Isomorphism Theorem for models of uncountable regular power (see [2]). We do obtain the following interpolation theorem generalizing Corollary 2 of $\S 2 . L^{p}(\lambda)$ is the set of all formulas equivalent to some $\left(Q_{\xi} v_{\xi}\right)_{\xi<\lambda} \chi$, where $\chi$ is quantifier-free. Hence every formula of $L_{\lambda+\lambda}$ belongs to $L^{p}(\lambda)$.

THeOREM 6. Let $L_{1}$ and $L_{2}$ be languages, with at most $\lambda^{\lambda}$ nonlogical symbols, whose intersection is $L$. Assume that $\theta \in L_{1}^{p}(\lambda), \psi \in L_{2}^{p}(\lambda)$, and $\vDash \theta \rightarrow \neg \psi$. Then there is some $\sigma \in L^{p}\left(\lambda^{\lambda}\right)$ such that $\vDash \theta \rightarrow \sigma$ and $\vDash \sigma \rightarrow \neg \psi$.

The case where $\theta$ and $\psi$ belong to $L_{i_{\lambda}{ }_{\lambda}}$ was proved (syntactically) by Takeuti [4], in response to Malitz's examples of implications which have no interpolants in any $L_{\kappa \lambda}$. This case in fact implies the above form of the theorem, but Takeuti does not obtain the general results from which we derive it.

\section{REFERENCES}

1. C. C. Chang, "Some remarks on the model theory of infinitary languages," in The syntax and semantics of infinitary languages, Springer-Verlag, Berlin and New York, 1968, pp. 36-63.

2. H. J. Keisler, "Formulas with linearly ordered quantifiers," in The syntax and semantics of infinitary languages, Springer-Verlag, Berlin and New York, 1968, pp. 96-130.

3. L. Svenonius, On the denumerable models of theories with extra predicates, Proc. Internat. Sympos. Theory of Models (Berkeley, 1963), North-Holland, Amsterdam, 1965, pp. 376-389. MR 35 \#42.

4. G. Takeuti, "A determinate logic," in The syntax and semantics of infinitary languages, Springer-Verlag, Berlin and New York, 1968, pp. 237-264.

5. A. Tarski, Remarks on predicate logic with infinitely long expression, Colloq. Math. 6 (1958), 171-176. MR 20 \#6351.

Department of Mathematics, University of Michigan, Ann Arbor, Michigan 48104 